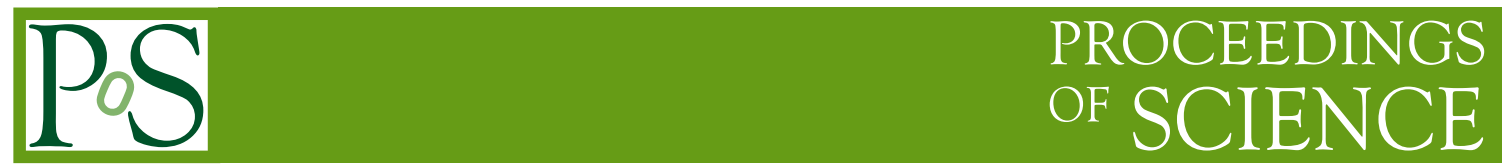

\title{
Power counting ans scaling for tensor models
}

\section{Thomas Krajewski* ${ }^{*}$}

Centre de Physique Théorique, Aix-Marseille Université, France

E-mail: thomas.krajewskiecpt.univ-mrs.fr

\section{Reiko Toriumi}

Department of Physics, University of California Berkeley, USA

E-mail: torirei@gmail.com

Random tensors are natural generalisations of matrix models related to random geometries of dimension $D$. Here, we revisit the large $N$ limit of tensor models and the power counting of tensorial group field theories using a renormalisation group equation.

Proceedings of the Corfu Summer Institute 2015 "School and Workshops on Elementary Particle Physics and Gravity"

1-27 September 2015

Corfu, Greece

\footnotetext{
* Speaker.

†Partially supported by the ANR grant "CombPhysMat2Tens".
} 
Despite decades of efforts, the construction of a quantum theory of gravity remains an open question. There are a few promising approaches, including string theory, loop quantum gravity, simplicial quantum gravity, ... Random tensors are higher dimensional generalisations of matrix models that have been introduced [1] to reproduce in dimension $D>2$ the successes of matrix models in two dimensional quantum gravity.

However, the combinatorics of tensor models is more intricate than the matrix model one. Progress in the field remained very slow until the advent of colored models [2]. Since then, many results have been obtained. We refer, among other reviews, to the series "Tensor track" [3], that retraces the development of colored models and its relation to quantum gravity.

Among these important results, let us mention the large $N$ limit [4] and Gaussian universality [5], as well as the construction of renormalisable models first in the abelian case [6], extended to the non abelian case [7]., Here, we revisit these results in the light of Polchinski's exact renormalisation group [8], suitably adapted to tensor models. It is based on results that appeared in [9], see also [10] and [11].

\section{Random tensors}

Random tensors are natural generalisations of random matrices that generate sums over random higher dimensional geometries. In dimension $D$, we consider a rank $D$ tensors $T_{i_{1}, \ldots, i_{D}}$ which corresponds to a simplex of dimension $D-1$, see figure 1 .

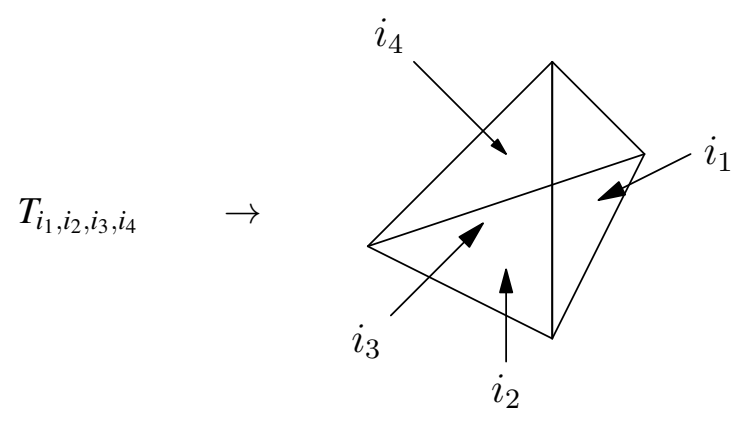

Figure 1: A random tensor in $D=4$ and the associated tetrahedron

The tensors we consider are complex and we treat $T$ and $\bar{T}$ as independent variables. Moreover, we do not impose and permutational symmetry between the indices of the tensors. This formalism is obtained from the original colored models [2] after integration over some of the degrees of freedom [12].

Our aim is to study the "path integral" over tensors,

$$
\log Z=\log \int d \bar{T} d T \exp \left\{-\bar{T} \cdot C^{-1} \cdot T+V_{0}(\bar{T}, T)\right\}=\sum_{\substack{\text { Feynan graph } \mathscr{G} \Leftrightarrow) \\ \text { dimension } D \text { triangulation }}} \mathscr{A} \mathscr{G}
$$

whose perturbative expansion yields a sum over a random triangulated geometries of dimension $D$, weighted by an amplitude $\mathscr{A} \mathscr{G}$. These geometries are constructed by gluing together the simplices represented by the tensors using the quadratic part of the action. The latter involves a propagator 
which is diagonal in the tensor indices

$$
C_{i_{1} \ldots i_{D} \bar{i}_{1} \ldots \bar{i}_{D}}=t \delta_{i_{1} \bar{i}_{1}} \cdots \delta_{i_{D} \bar{i}_{D}}
$$

with $t$ a positive parameter. Then, Wick's theorem amounts to gluing the tetrahedra corresponding to $T$ and $\bar{T}$ together, see figure 2 . To determine a suitable form for the interacting potential, we
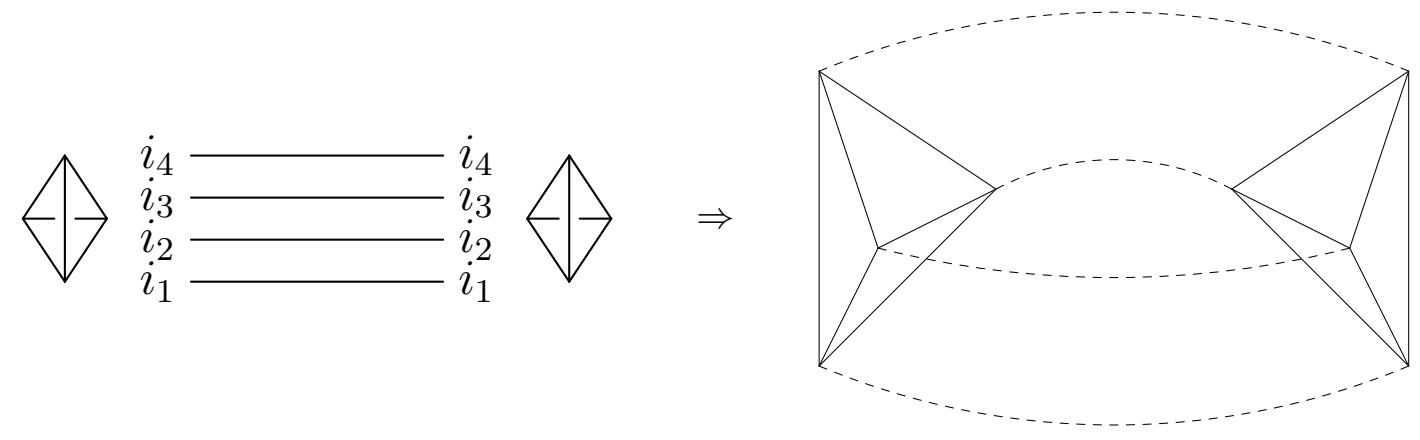

Figure 2: Gluing two tetrahedra using a propagator

impose that the latter be invariant under the unitary transformations,

$$
T_{i_{1}, \ldots, i_{D}} \rightarrow \sum_{j_{1}, \ldots, j_{D}} U_{i_{1} j_{1}}^{1} \cdots U_{i_{D} j_{D}}^{D} T_{j_{1}, \ldots, j_{D}}, \quad \bar{T}_{i_{1}, \ldots, i_{D}} \rightarrow \sum_{j_{1}, \ldots, j_{D}} \bar{U}_{i_{1} j_{1}}^{1} \cdots \bar{U}_{i_{D} j_{D}}^{D} \bar{T}_{j_{1}, \ldots, j_{D}},
$$

with $\left(U^{1}, \ldots, U^{D}\right) \in \mathrm{U}(N) \times \cdots \times \mathrm{U}(N)$. Any invariant potential can be expanded over graphs called $D$-bubbles. A $D$-bubble is a bipartite $D$-colored graph, not necessarily connected. This means that:

- There are two types of vertices, black ones $\bullet$ and white ones $\circ$.

- An edge can connect only a black vertex to a white vertex.

- At any vertex there are exactly $D$ incident edges.

- Each edge is decorated by a color in $\{1, \ldots, D\}$ in such a way that the colors of the $D$ edges incident to any vertex are all different.

The invariant associated to a $D$-bubble is defined by assigning a tensor $T$ to a white vertex, a tensor $\bar{T}$ to a black vertex, identify the indices $i_{k}$ in $T$ and $\bar{i}_{k}$ in $\bar{T}$ whenever they are connected by a line of color $k$ and summing over all tensor indices. In analogy with the trace invariants of matrix models to which it reduces for $D=2$, such an invariant is written as

$$
\operatorname{Tr}_{\mathscr{B}}(T, \bar{T})=
$$

$$
\sum_{\substack{i_{e} \\ \text { edge indices }}} \prod_{\text {black vertices }} \bar{T}_{\bar{i}_{\bar{v}, 1}, \ldots, \bar{i}_{\bar{v}, D}} \prod_{\text {white vertices }} T_{i_{v, 1}, \ldots, i_{v, D}} \prod_{\substack{e \\ \text { edges }}} \delta_{i_{e}, \bar{i}_{\bar{v}}(e), c(e)} \delta_{e_{e}, i_{V_{0}}(e), c(e)},
$$

where $e$ is an edge between a white vertex $V_{0}(e)$ and a black vertex $\bar{v}(e)$ and $c(e)$ its color. For example, the invariants associated to two 3-bubbles are given in figure 3. 


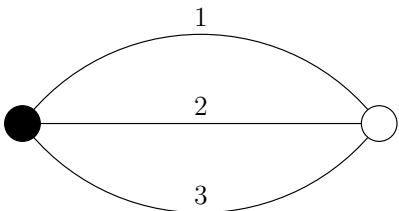

$$
\sum_{i_{a}} \bar{T}_{i_{1}, i_{2}, i_{3}} T_{i_{1} i_{2} i_{3}}
$$

(a) Dipole graph (Gaußian measure)

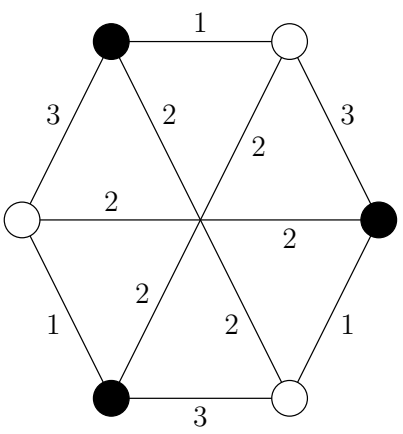

$$
\sum_{i_{a}, j_{b}, k_{c}} \bar{T}_{i_{1} i_{2} i_{3}} \bar{T}_{j_{1} j_{2} j_{3}} \bar{T}_{k_{1} k_{2} k_{3}} T_{i_{1} k_{2} j_{3}} T_{j_{1} i_{2} k_{3}} T_{k_{1} j_{2} i_{3}}
$$

(b) Degree 6 interaction

Figure 3: Some bubble invariants

Then, the potential is expanded as

$$
V_{0}(T, \bar{T})=\sum_{\mathscr{B}} \frac{\lambda_{\mathscr{B}}}{C_{\mathscr{B}}} \operatorname{Tr}_{\mathscr{B}}(\bar{T}, T) .
$$

with $\lambda_{\mathscr{B}}$ a coupling constant and $C_{\mathscr{B}}$ the order of the group of transformations preserving the bubble. From a geometrical point of view, any bubble encodes a triangulation of dimension $D-1$ obtained by gluing together simplices of dimension $D-1$. They are the basic building blocks of the dimension $D$ triangulations in (1.1).

Finally, let us mention that beyond the partition function $Z$ in (1.1), it may also be of interest to compute the expectation value of some observables

$$
\langle\mathscr{O}\rangle=\frac{\int d \bar{T} d T \mathscr{O}(T, \bar{T}) \exp \left\{-\bar{T} \cdot C^{-1} \cdot T+V_{0}(\bar{T}, T)\right\}}{Z} .
$$

Invariant observables can be also expressed in terms of bubble invariants.

\section{Large $N$ limit and melonic universality}

In the case of matrix models, it is well known that the large $N$ expansion leads to inverse powers of the genus $g$ of the triangulated surface

$$
\frac{1}{N^{2}} \log Z=\frac{1}{N^{2}} \log \int d \bar{M} d M \exp \left\{-N \bar{M} \cdot C^{-1} \cdot M+N V_{0}(\bar{M}, M)\right\}=\sum_{\substack{\text { Feynan graph } \mathscr{G} \Leftrightarrow \\ \text { triangulated surface }}} \frac{1}{N^{2 g(\mathscr{G})}} \mathscr{A} \mathscr{G} .
$$

The genus $g \geq 0$ is defined through the Euler caracteristics

$$
2-2 g=\#\{\text { vertices }\}-\#\{\text { edges }\}+\#\{\text { faces }\} .
$$


A similar result holds for random tensors, see the work by Gurau [4]. Here, we derive this result from a renormalisation group equation.

The alluded renormalisation group equation is a tensorial version of Polchinski's equation, initially formulated in the context of field theory in [8]. In our context, it is a differential equation for the effective potential $V_{t}(T, \bar{T})$ defined by

$$
V_{t}[T, \bar{T}]=\log \int \frac{d \bar{T}^{\prime} d T}{\mathscr{N}_{t}} \exp \left\{-\bar{T} \cdot C_{t}^{-1} \cdot T+V_{0}\left[T+T^{\prime}, \bar{T}+\bar{T}^{\prime}\right]\right\},
$$

with $C_{t}$ the $t$ dependent propagator as in (1.2) and $\mathscr{N}_{t}$ a trivial normalisation factor. Note that in this simple context, $t$ cannot be interpreted as a cut-off but is merely a parameter that controls the flow. It interpolates between $t=0$ (no integration) and $t=1$ (complete integration).

Following the partial integration technique presented in [13], the effective potential obeys the differential equation

$$
\frac{\partial V}{\partial t}=\frac{\partial^{2} V}{\partial T \partial \bar{T}}+\frac{\partial V}{\partial T} \frac{\partial V}{\partial \bar{T}}
$$

This equation is represented diagrammatically in figure 4 . We refer to the first term as the loop term and the second one as the tree term. At a geometrical level for $D=3$, this equation is pictured in figure 5

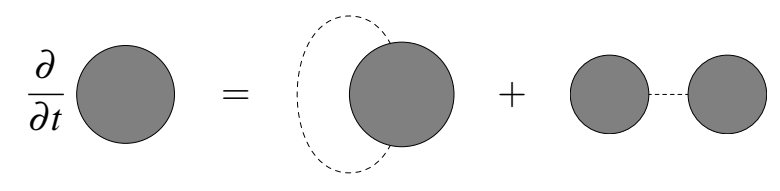

Figure 4: Diagrammatic representation of Polchinski's equation

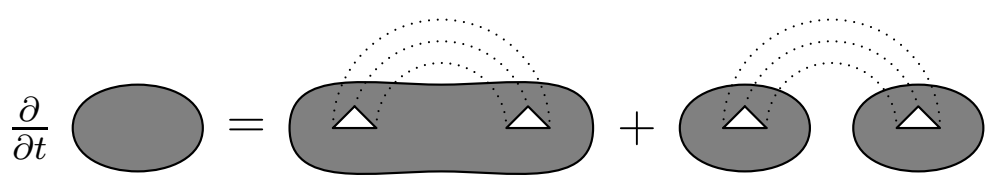

Figure 5: Geometrical interpretation of Polchinski's for rank 3 tensors

In order to translate equation (2.4) into a system of equations for the couplings $\lambda_{\mathscr{B}}$, it is helpful to introduce the notion of a cut. A $k$-cut $c$ (for $k \in\{0,1, \ldots, D\}$ ) in a $D$-bubble $\mathscr{B}$ is defined as a subset of $k$ edges $\left\{e_{1}, \ldots, e_{k}\right\}$ of $\mathscr{B}$ with different colors. The cut bubble $\mathscr{B}_{c}$ is the bubble obtained from $\mathscr{B}$ by cutting the $k$ edges $\left\{e_{1}, \ldots, e_{k}\right\}$ into half-edges, attaching to them a new black $\bar{v}$ and a new white vertex $v$ and joining $v$ and $\bar{v}$ by $D-k$ edges carrying the colors not in $\left\{e_{1}, \ldots, e_{k}\right\}$. This ensures that $\mathscr{B}_{c}$ is a bubble with $D$ colors. In particular, if $c$ is a 0 -cut, $\mathscr{B}_{c}$ is just the disjoint union of $\mathscr{B}$ with a dipole. A 1 -cut on an edge $e$ is just the insertion on $e$ of a pair of vertices joined by $D-1$ edges carrying the colors different from that of $e$. Then, the flow equation for the bubble couplings writes

$$
\frac{\partial \lambda_{\mathscr{B}}}{\partial t}=\sum_{k=0}^{D} \sum_{k \text {-cut } c} N^{D-k} \lambda_{B_{c}}+\sum_{\substack{D \text {-cut } c \\ \kappa\left(\mathscr{B}_{C}\right)>\kappa(\mathscr{B})}} \sum_{\mathscr{B}_{c}=\mathscr{B}^{\prime} \cup \cup \mathscr{B}^{\prime \prime}, v \in \mathscr{B}^{\prime \prime}, \overline{\mathscr{B}^{\prime}, \overline{B^{\prime \prime}}}} \lambda_{\mathscr{B}^{\prime}} \lambda_{\mathscr{B}^{\prime \prime}}
$$



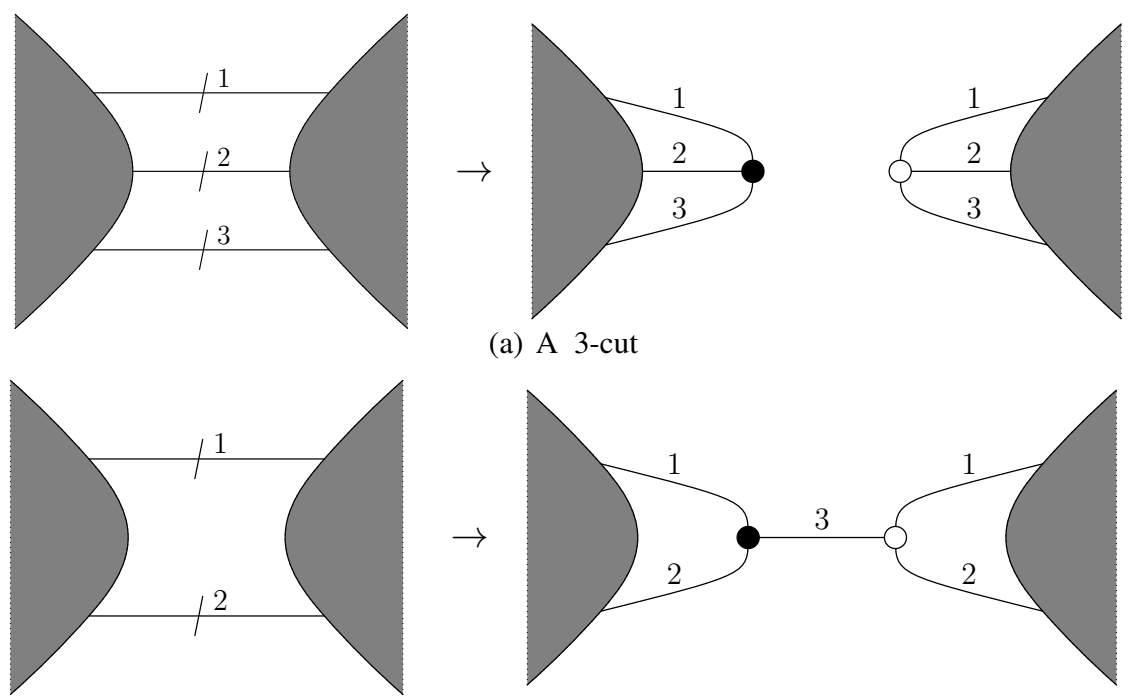

(a) A 3-cut

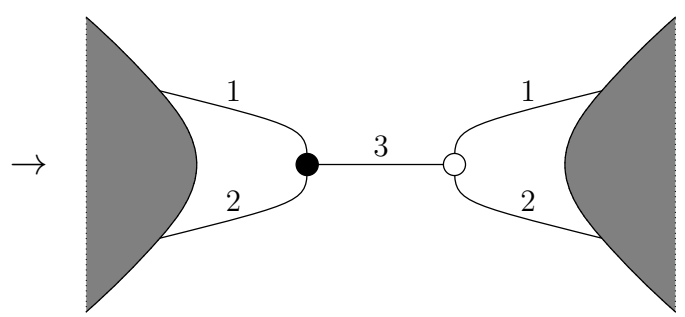

(b) A 2-cut

Figure 6: Examples of cut operations

In this equation, the sum runs over $k$-cuts $c$ for $0 \leq k \leq D$. The second term involves a summation over $D$-cuts that increases the number of connected components $\kappa(\mathscr{B})$ of $\mathscr{B}$ and over ways of writing $\mathscr{B}_{c}$ as a disjoint union of a bubble $\mathscr{B}^{\prime}$ containing $v$ and a bubble $\mathscr{B}^{\prime \prime}$ containing $\bar{v}$. Finally, let us emphasize that even if the initial potential $V_{0}$ does not contain non connected bubbles, the latter are generated by the loop-like term in the flow equation.

This system of differential equations for the bubble couplings $\lambda_{\mathscr{B}}$ involves positive powers of $N$ and is therefore not suitable for a large $N$ limit. It is helpful to introduce the analogue of dimensionless couplings $u_{\mathscr{B}}$ in quantum field theory, defined by

$$
\lambda_{\mathscr{B}}=N^{\delta(\mathscr{B})} u_{\mathscr{B}}
$$

The scaling dimension of the bubble is defined as

$$
\delta(\mathscr{B})=\mathscr{D}-\kappa(\mathscr{B})
$$

with $\kappa(\mathscr{B})$ the number of connected components of $\mathscr{B}$. Similarly, we rescale the propagator as $C \rightarrow \frac{C}{N^{D-1}}$.In terms of these new variables, the system of differential equations reads

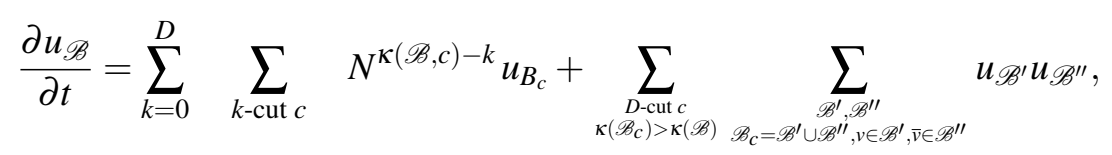

where $\kappa(\mathscr{B}, c)$ is the number of connected components of $\mathscr{B}$ containing edges of the cut, except for a $D$-cut that increases the number of connected components of $\mathscr{B}$, in which case $\kappa(\mathscr{B}, c)=0$. It allows to relate the number of connected components of $\mathscr{B}_{c}$ and of $\mathscr{B}$ as $\kappa\left(\mathscr{B}_{c}\right)=\kappa(\mathscr{B})-$ $\kappa(\mathscr{B}, c)+1$. Since $\kappa(\mathscr{B}, c) \leq k$, there are only non positive powers of $N$ in (2.8). We give a few example for low order bubble couplings in appendix A.

The system (2.8) can be solved iteratively at any finite order in $t$ to express the bubble couplings $u_{\mathscr{B}}(t)$ in terms of the bubble couplings $u_{\mathscr{B}}(0)$, with only non positive powers of $N$. Identifying the 
bubble coupling $u_{\emptyset}(1)$ with $\frac{\log Z}{N^{D}}$, we arrive at a tensor model analogue of the large $N$ matrix model expansion (2.1),

$$
\begin{aligned}
\frac{1}{N^{D}} \log Z & =\frac{1}{N^{D}} \log \int d \bar{T} d T \exp \left\{-N^{D-1} \bar{T} \cdot C^{-1} \cdot T+\sum_{\mathscr{B}} \frac{N^{D-\kappa(\mathscr{B})} u_{\mathscr{B}}(0)}{C_{\mathscr{B}}} \operatorname{Tr}_{\mathscr{B}}(\bar{T}, T)\right\} \\
& =\sum_{\substack{\text { Feyman graph } \mathscr{G} \Leftrightarrow \\
\text { dimension D triangulation }}} \frac{1}{N^{\omega(\mathscr{G})}} \mathscr{A}_{\mathscr{G}}
\end{aligned}
$$

where $\omega$ is a positive integer called the degree.

Moreover, if we are interested in the terms in (2.8) that survive in the limit $N \rightarrow \infty$, we only consider contributions in the loop term such that $\kappa(\mathscr{B}, c)=k$. This means that we can perform 0 -cuts or $k$-cuts with at most one cut edge in each connected component. Starting with the empty bubble (corresponding to $\log Z$ ), we obtain the dipole with a 0 -cut. Then, a repeated application of the allowed cuts and of cuts that disconnect the bubbles in the tree term only generate melonic bubbles $\mathscr{M}$. A bubble is melonic if, for every white vertex $v$, there is a black vertex $\bar{v}$ such that removing of $v$ and $\bar{v}$ increases the number of connected components by $D-1$. A few examples of melonic and non melonic bubbles are given in figure 7. Consequently, this leads to melonic universality: $\lim _{N \rightarrow \infty} \frac{\log Z}{N^{D}}$ only depend on the melonic couplings $u_{\mathscr{M}}(0)$. This result extends to the expectation value of observables and it may be shown that the result can be computed in a Gaußian theory [9]. Thus, we have a alternative proof, based on the renormalisation group equation, of Gurau's universality result [5].

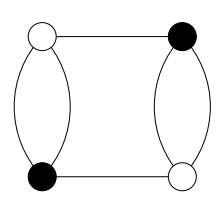

(a) Melonic bubbles

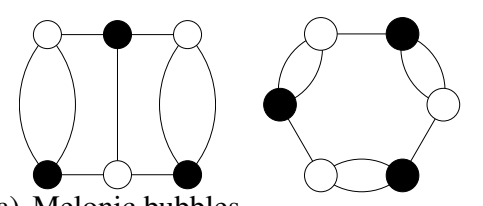

Figure 7: Melonic and non meloinc bubbles for $D=3$ and $D=4$

\section{Group field theories}

In dimension $D$, group field theories are quantum field theories defined on $D$ copies of a group $G$ whose interactions are modelled on those of tensor models. Their perturbative expansion leads to a sum over triangulations, weighted by a spin foam amplitude,

$$
\log Z=\log \int[d \bar{\Phi}][d \Phi] \exp \left\{-\bar{\Phi} \cdot C^{-1} \cdot \Phi+V_{0}(\bar{\Phi}, \Phi)\right\}=\sum_{\substack{\text { Feynan graph } \mathscr{G} \Leftrightarrow \\ \text { dimension } D \text { triangulation }}} \mathscr{A}_{\mathscr{G}}^{\text {spin foam }}
$$

Spin foams amplitudes are space-time transition amplitudes between loop quantum gravity states. We refer the reader to the monographs [14] and [15] for some background on loop quantum gravity, spin foams and group field theories. Moreover, the reviews [16], [17] and [18] present some general facts on group field theories. Models of interest in quantum gravity involve the groups $\mathrm{SU}(2)$ (Euclidian $D=3$ ), $\mathrm{SO}(4)$ (Euclidian $D=4$ ) and $\mathrm{SL}(2, \mathrm{C})$ (Lorentzian $D=4$ ). 
Here we work with tensorial group field theories. The group field is a complex one and we do not impose any permutational symmetry of its argument. It is constructed in analogy with tensors

$$
T_{i_{1}, \ldots, i_{D}} \rightarrow \Phi\left(g_{1}, \ldots, g_{D}\right) \quad \bar{T}_{i_{1}, \ldots, i_{D}} \rightarrow \Phi\left(\bar{g}_{1}, \ldots, \bar{g}_{D}\right) .
$$

$\bar{g}$ is not the complex conjugate of $g$ but an independent group element that is an argument of the complex conjugate field $\bar{\Phi} . \Phi\left(g_{1}, \ldots, g_{D}\right)$ represents a $D-1$ simplex and the group variables $g_{1}, \ldots, g_{D}$ are associated with its $D(D-1)$-faces and can be interpreted as parallel transporters, see figure 9 .

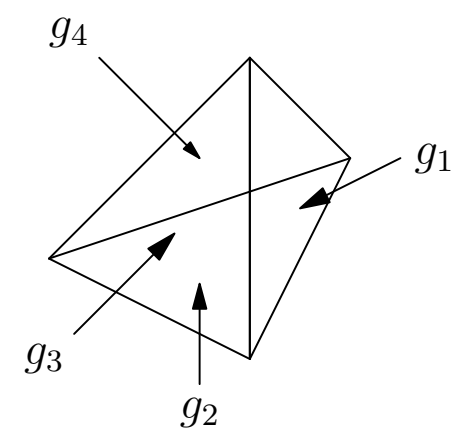

Figure 8: The group field as a tetrahedron.

The group field is assumed to obey a closure condition, related to the gauge invariance of the model,

$$
\Phi\left(g_{1}, \ldots, g_{D}\right)=\Phi\left(h g_{1}, \ldots, h g_{D}\right), \quad \bar{\Phi}\left(\bar{g}_{1}, \ldots, \bar{g}_{D}\right)=\bar{\Phi}\left(\bar{h} \bar{g}_{1}, \ldots, \bar{h} \bar{g}_{D}\right) .
$$

for every group elements $h$ and $\bar{h}$. The propagator is constructed using the heat kernel on the group $H_{\alpha}$,

$$
C_{\Lambda, \Lambda_{0}}\left(\left\{g_{e} \bar{g}_{e}^{-1}\right\}\right)=\int_{\frac{1}{\Lambda_{0}^{2}}}^{\frac{1}{\Lambda^{2}}} d \alpha \int d h d \bar{h} \prod_{1 \leq i \leq D} H_{\alpha}\left(h g_{i} \bar{g}_{i}^{-1} \bar{h}^{-1}\right),
$$

with $\Lambda$ an IR cut-off and $\Lambda_{0}$ a UV cut-off. Group integrations are performed using the Haar measure and implement the required invariances. As for tensor models, it induces the gluing of $(D-1)$ simplices, see figure 9, but now there is a non trivial separation between slow and fast modes, as is usually the case in quantum field theory.

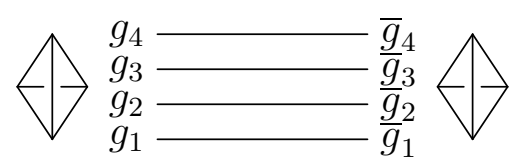

Figure 9: Propagation of a tetrahedron.

We expand the interacting potential over bubble couplings as

$$
\begin{aligned}
V(\Lambda, \Phi, \bar{\Phi})=\sum_{\mathscr{B}} & \frac{1}{C_{\mathscr{B}}} \int \prod_{e} d g_{v(e), c(e)} d \bar{g}_{\bar{v}(e), c(e)} \\
& \lambda_{\mathscr{B}}\left(\Lambda,\left\{g_{v(e), c(e)}\left(\bar{g}_{\bar{v}(e), c(e)}\right)^{-1}\right\}\right) \prod_{\text {black vertices }} \Phi\left(\bar{g}_{\bar{v}, 1}, \ldots, \bar{g}_{\bar{v}, D}\right) \prod_{v} \Phi\left(g_{v, 1}, \ldots, g_{v, D}\right)
\end{aligned}
$$


where $\delta$ is the Dirac distribution on the group, defined by $\int d g \delta(g) f(g)=f(1)$. Note that we have imposed translation invariance, so that the couplings only depend on the products $g_{v(e), c(e)}\left(\bar{g}_{\bar{v}(e), c(e)}\right)^{-1}$

\section{Power counting for Abelian models with closure cosntraint}

Although the analogue of Polchinski's equation can be formulated for a general group field theory [9], we consider in this section only abelian models with group $G=\mathrm{U}(1)^{d}$. Besides being technically much easier, this allows us to have a general formula depending on the dimension of the group $d$ and the rank of the field $D$, to be identified with the space-time dimension. Elements of $\mathrm{U}(1)$ are paramterised as $g=\exp 2 \mathrm{i} \pi \frac{\theta}{L}$ with $\theta \in[0, L]$ where $L$ being a length. The heat kernel covariance is

$$
C\left(\Lambda, \Lambda_{0},\left\{\theta_{i}-\bar{\theta}_{i}\right\}\right)=\int_{\frac{1}{\Lambda_{0}^{2}}}^{\frac{1}{\Lambda^{2}}} d \alpha \sum_{\left\{p_{i}\right\} \in \frac{1}{L} \mathbb{Z}^{d D}} \exp -\left\{\alpha \sum_{1 \leq i \leq D} p_{i}^{2}+\mathrm{i} \sum p_{i}\left(\theta_{i}-\bar{\theta}_{i}\right)\right\} \delta_{\sum p_{i}, 0}
$$

where we have enforced the condition $\sum_{i} p_{i}=0$ so that the closure constraint is fulfilled. Since the group is compact, the momenta $p_{i} \in \frac{1}{L} \mathbb{Z}^{d}$ are discrete.

Formulated in momentum space, the system of differential equation for the bubble couplings derived from Polchinski's equation is

$$
\begin{aligned}
& \Lambda \frac{\partial \lambda_{\mathscr{B}}\left(\left\{p_{e}\right\}\right)}{\partial \Lambda}=-\frac{2}{\Lambda^{2}} \sum_{k=0}^{D} \sum_{k \text {-cut } c} \sum_{\left\{p_{l}\right\}_{l \notin c}} \exp -\frac{1}{\Lambda^{2}}\left\{\sum_{i=1}^{D} p_{i}^{2}\right\} \lambda_{B_{c}}\left(\left\{p_{e}\right\}_{e \in \mathscr{B}},\left\{p_{l}\right\}_{l \notin c}\right) \delta_{\sum_{i=1}^{D} p_{i}, 0}
\end{aligned}
$$

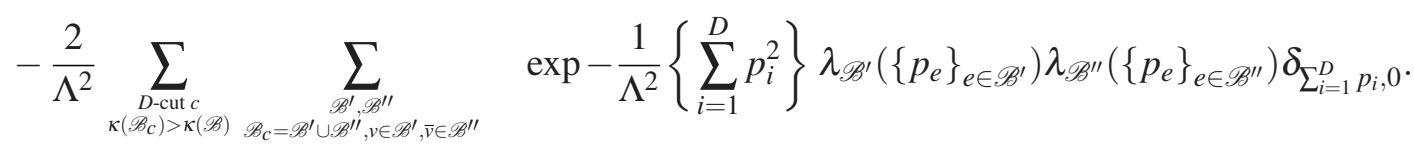

It is the group field theory analogue of Polchinski's quantum field theory equation written for correlation functions in momentum space. Because of the closure constraint, there is a momentum conservation at every vertex of the bubbles. Note that $\delta_{\sum_{i=1}^{D} p_{i}, 0}$ can be omitted in the tree term since this condition is already satisfied by the couplings. In the loop term, it reduces to $\delta_{\sum_{i \in c} p_{i}, 0}$, with a sum reduced to momenta in the cut. If $L$ is large enough $(1 / L \ll \Lambda)$ momenta can be treated as continuous variables. Then, we replace sums by integrals

$$
\sum_{p} \rightarrow L^{d} \int d p \quad \text { and } \quad \delta_{\sum p_{i}, 0} \rightarrow L^{-d} \delta\left(\sum p_{i}\right)
$$

To investigate the large $\Lambda$ behaviour, we introduce the analogue of dimensionless variables as

$$
\lambda_{\mathscr{B}}\left(\left\{p_{e}\right\}, \Lambda\right)=\Lambda^{\delta(\mathscr{B})} u_{\mathscr{B}}\left(\left\{q_{e}\right\}, \Lambda\right) \quad \text { with } \quad p_{e}=\Lambda q_{e}
$$


$\delta(\mathscr{B})$ is a scaling dimension in the large $\Lambda$ limit that that can be determined from the flow equation,

$$
\begin{aligned}
& \Lambda \frac{\partial u_{\mathscr{B}}\left(\left\{q_{e}\right\}\right)}{\partial \Lambda}=-\delta(\mathscr{B}) u_{\mathscr{B}}\left(\left\{q_{e}\right\}\right)+\sum_{e} q_{e} \frac{\partial u_{\mathscr{B}}\left(\left\{q_{e}\right\}\right)}{\partial q_{e}} \\
& -2 \sum_{k=0}^{D} \sum_{\substack{0 \leq k \leq D \\
c k \text { cut }}} \frac{\Lambda^{\delta\left(\mathscr{B}_{c}\right)-\delta(\mathscr{B})-2+d(D-k)-d}}{L^{d-d(D-k)}} \int \prod_{l \notin c} d q_{l} \exp -\left(\sum_{i} q_{i}^{2}\right) u_{B_{c}}\left(\left\{q_{e}\right\}_{e \in \mathscr{B}},\left\{q_{l}\right\}_{l \notin c}\right) \delta\left(\sum_{i \in c} q_{i}\right) \\
& -2 \sum_{\substack{D \text {-utc } c \\
\kappa(\mathscr{B} c)>(\mathscr{B})}} \sum_{\substack{\mathscr{B}_{c}=\mathscr{O}^{\prime}, \cup \mathscr{B}^{\prime \prime}, v \in \mathscr{B}^{\prime}, \bar{v} \in \mathscr{B}^{\prime \prime}}} \frac{\Lambda^{\delta\left(\mathscr{B}^{\prime}\right)+\delta\left(\mathscr{B}^{\prime \prime}\right)-\delta(\mathscr{B})-2}}{L^{d}} \exp -\left(\sum_{i} q_{i}^{2}\right) u_{\mathscr{B}^{\prime}}\left(\left\{q_{e}\right\}_{e \in \mathscr{B}^{\prime}}\right) u_{\mathscr{B}^{\prime \prime}}\left(\left\{q_{e}\right\}_{e \in \mathscr{B}^{\prime \prime}}\right) .
\end{aligned}
$$

In analogy with the tensor model case, let us assume that $\delta(\mathscr{B})$ can be determined in such a way that the flow equation only involves non positive powers of $\Lambda$,

$$
\Lambda \frac{\partial u(\mathscr{B})}{\partial \Lambda}=\beta_{0}\left(u\left(\mathscr{B}^{\prime}\right)\right)+\frac{1}{\Lambda} \beta_{1}\left(u\left(\mathscr{B}^{\prime}\right)\right)+\frac{1}{\Lambda^{2}} \beta_{0}\left(u\left(\mathscr{B}^{\prime}\right)\right)+\ldots
$$

In quantum field theory, the variables $u_{\mathscr{B}}$ would be truly dimensionless variables and we only have $\beta_{0}$. In our context this is not possible, since $\delta(\mathscr{B})$ is only a scaling dimension for large $\Lambda$, not the canonical dimension related to the rescaling of both $\Lambda$ and $L$. A similar phenomenon has been first noted in [19] in a similar context. Nevertheless, the negative powers of $\Lambda$ are harmless as far as we are interested in the UV regime.

To determine the scaling dimensions, let us make the ansatz

$$
\delta(\mathscr{B})=\alpha+\beta \kappa(\mathscr{B})+\gamma v(\mathscr{B})
$$

where $\kappa(\mathscr{B})$ is the number of connected components and $v(\mathscr{B})$ the number of vertices. The exponent of $\Lambda$ in the tree term is

$$
\delta\left(\mathscr{B}^{\prime}\right)+\delta\left(\mathscr{B}^{\prime \prime}\right)-\delta(\mathscr{B})-2=\alpha+\beta+2 \gamma-2
$$

while for the loop term it is

$$
\delta\left(\mathscr{B}_{c}\right)-\delta(\mathscr{B})-2+d(D-k)-d=-d k-\beta \kappa(\mathscr{B}, c)+\beta+2 \gamma-2+d(D-1),
$$

where we recall that $\kappa(\mathscr{B}, c)$ is the number of connected components of $\mathscr{B}$ containing edges of the cut and vanishes for a $D$-cut that disconnects the graph. It obeys $\kappa\left(\mathscr{B}_{c}\right)=\kappa(\mathscr{B})-\kappa(\mathscr{B}, c)+1$. Setting $\alpha=d(D-1), \beta=-d$, and $2 \gamma=-(d(D-2)-2)$, the exponent of the tree term vanishes while for the loop term it is $d(\kappa(\mathscr{B}, c)-k)$, therefore always negative. Consequently, with a scaling dimension

$$
\delta(\mathscr{B})=d(D-1)-d \kappa(\mathscr{B})-(d(D-2)-2) \frac{v(\mathscr{B})}{2},
$$

the couplings $u_{\mathscr{B}}$ obey a flow equation with non positive powers of $\Lambda$. This scaling dimension reduces to the one found in [7] by Carrozza, Oriti and Rivasseau using multiscale analysis for the connected bubble $\kappa(\mathscr{B})=1$. 
In the Wilsonian picture, interactions with $\delta(\mathscr{B})>0$ are relevant, with $\delta(\mathscr{B})=0$ marginal and with $\delta(\mathscr{B})<0$ irrelevant. In perturbation theory, relevant and marginal interactions are renormalisable, in the sense that we may choose their couplings to depend on $\Lambda_{0}$ in such a way that all quantities of physical interest remain finite in the limit $\Lambda_{0} \rightarrow \infty$.

Let us close by listing all the renormalisable interactions for Abelian models with closure constraints. If we require $\delta(\mathscr{B}) \geq 0$, then $v(\mathscr{B}) \leq 2+\frac{4}{d(D-2)-2}$. Interactions involve bubble with at least 4 vertices, so that $d(D-2)=4$ or $d(D-2)=3$. As a consequence, we only have five renormalisable theories, as first discovered in [7] using multiscale analysis of Feynman graphs.

- $D=3$ and $d=4$ so that $\delta=8-4 \kappa-v$, with the unique renormalisable interaction

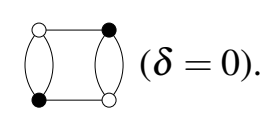

The fixed point structure of a non Abelian version of this model has been studied in [20] using the non Abelian group $\mathrm{SU}(2) \times \mathrm{U}(1)$.

- $D=4$ and $d=2$ so that $\delta=6-2 \kappa-v$ with only quartic renormalisable interactions

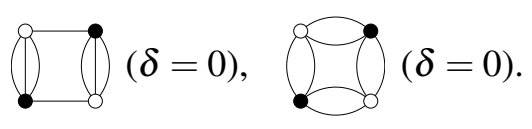

The second interaction is not melonic and called necklace in [21].

- $D=6$ and $d=1$ so that $\delta=5-\kappa-v$. The renormalisable interactions are quartic with melonic and non melonic interactions.

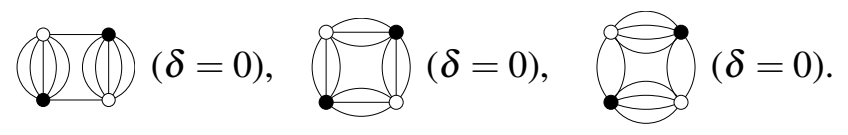

The model was shown to be renromalisable in [22] and [23] and its fixed point structure, was further investigated in [24] using a truncation of Wetterich's equation.

- $D=3$ and $d=3$ so that $\delta=6-3 \kappa-v / 2$. The renormalisable interactions are quartic and sextic

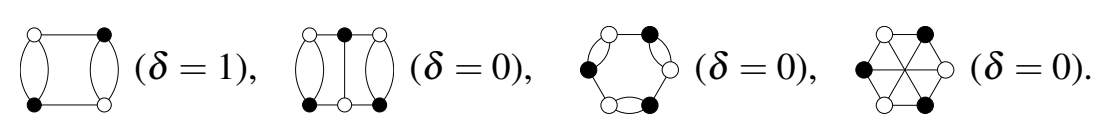

The first term is in fact superrenormalisable $(\delta>0)$ and the last one is not melonic. The non Abelian version with group $\mathrm{SU}(2)$ is the first non Abelian group field theory that has been renormalised [7]. It is related to $D=3$ quantum gravity.

- $D=5$ and $d=1$ so that $\delta=4-\kappa-v / 2$. Renormalisable interactions are quartic and sextic, with melonic interactions 


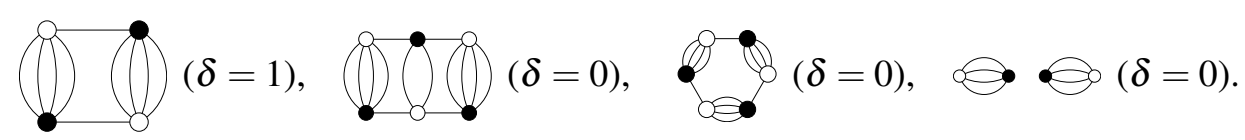

This model, including the non connected interaction, has been shown to be renormalisable in [22].

All these bubble couplings with $v \geq 4$ have dimension 0 or 1 , so that there is no possibility of adding a derivative coupling. A degree $n$ derivative would change the scaling dimension from $\delta(\mathscr{B})$ to $\delta(\mathscr{B})-n$. The case $n=1$ is excluded if we assume rotational symmetry.

Finally, let us emphasise that there are non melonic interactions among the renormalisable ones, which are usually set to 0 in the bare action. However, their actual behaviour under the renormalisation group equation is not known and certainly deserves more study.

\section{A. ERGE for low order bubble couplings}

In this appendix, a few examples of evolution equations for rescaled couplings are given.

\section{A.1 Couplings in rank $D=3$ tensors models}

$$
\begin{aligned}
\frac{\partial}{\partial t} u_{\odot} \bullet & {\left.\left[u_{\odot \bullet \bullet \bullet} \cdot\right]\right|_{0 \text { cut }}+\left.\left[3 u_{Q} \cdot\right]\right|_{1 \text { cut }}+\left.\left[u_{\odot}^{2} \cdot\right]\right|_{3 \text { cuts }} } \\
& +\left.\frac{1}{N}\left[3 u_{\odot} \cdot\right]\right|_{2 \text { cuts }}+\left.\frac{1}{N^{3}}\left[u_{\odot} \bullet \bullet \cdot\right]\right|_{3 \text { cuts }} .
\end{aligned}
$$

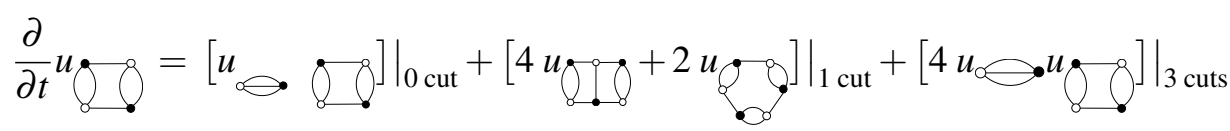

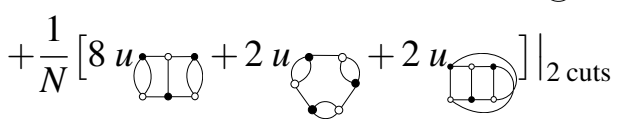

$$
\begin{aligned}
& +\left.\frac{1}{N^{2}}[4 u \leftarrow]\right|_{3 \text { cuts }}+\left.\frac{1}{N^{3}}[4 u \odot \cdot 8]\right|_{3 \text { cuts }} \text {. }
\end{aligned}
$$

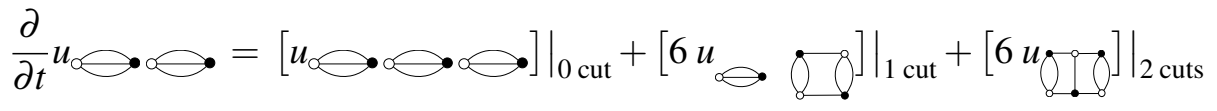

$$
\begin{aligned}
& +\left.\left[4 u_{\odot} \bullet \bullet \cdot u_{\odot} \cdot\right]\right|_{3 \text { cuts }} \\
& +\frac{1}{N}\left\{\left.\left[6 u_{\odot} \cdot-\cdot\right]\right|_{2 \text { cuts }}+\left.\left[6 u_{\bullet} \cdot\right]\right|_{3 \text { cuts }}\right\}+\left.\frac{1}{N^{3}}\left[2 u_{\odot} \bullet \bullet \bullet \bullet \cdot\right]\right|_{3 \text { cuts }} .
\end{aligned}
$$




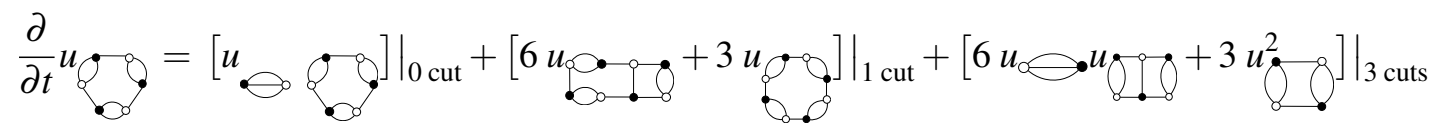

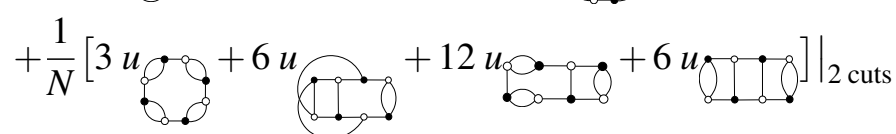

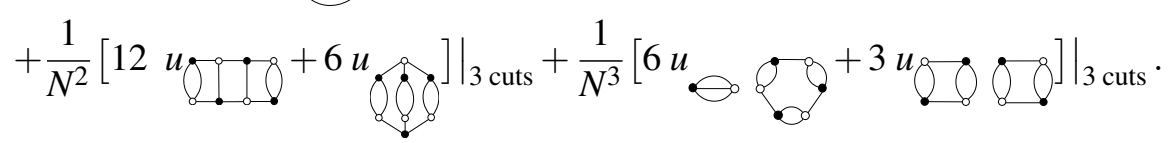

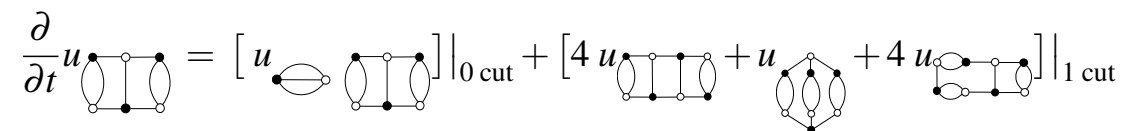

$$
\begin{aligned}
& +\left.\left[6 u_{\bullet} u_{\bullet} \cdot+2 u_{\bullet}^{2} \cdot\right]\right|_{3 \text { cuts }}
\end{aligned}
$$

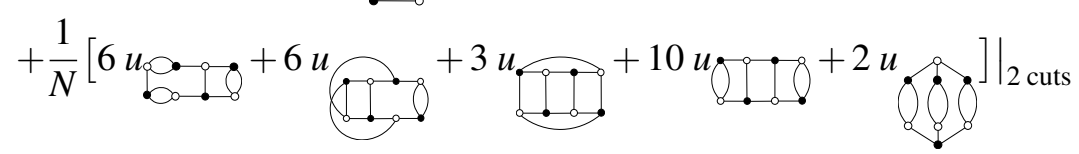

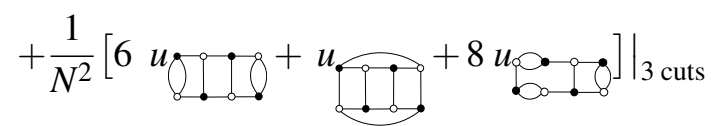

$$
\begin{aligned}
& +\left.\frac{1}{N^{3}}\left[6 u \leftrightarrow 0_{0}+2 u_{0} \cdot 0\right]\right|_{3 \text { cuts }}
\end{aligned}
$$

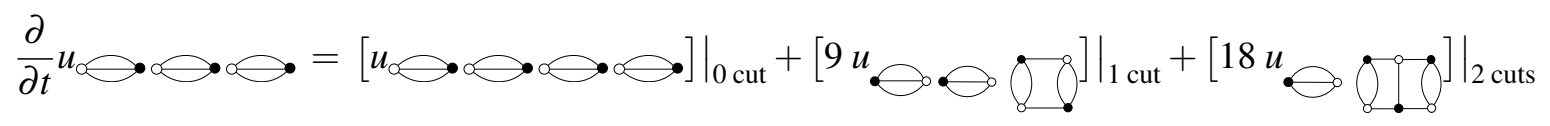

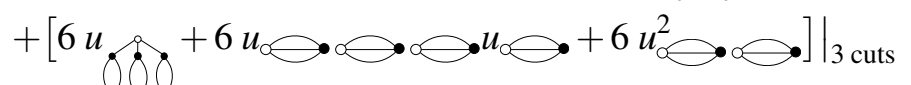

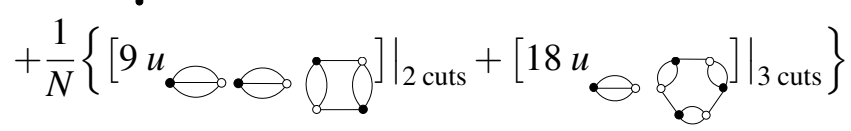

$$
\begin{aligned}
& +\frac{1}{N^{3}}\left[\left.3 u_{\odot} \bullet \bullet \bullet \bullet \cdot\right|_{3 \text { cuts }} .\right.
\end{aligned}
$$




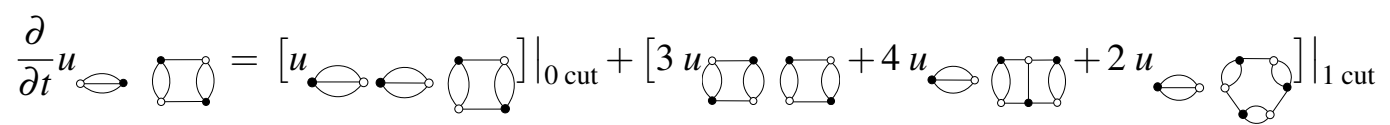

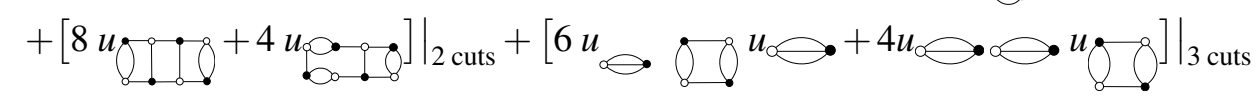

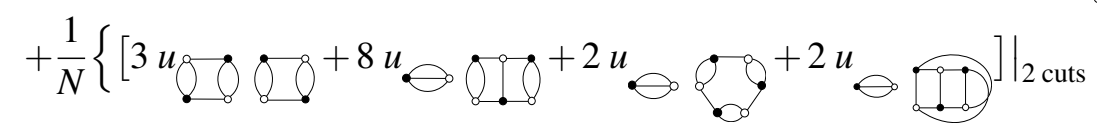

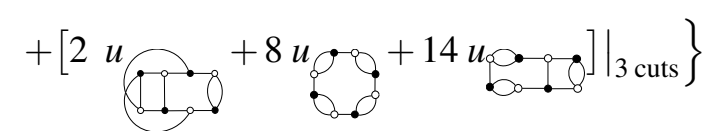

$$
\begin{aligned}
& +\left.\frac{1}{N^{2}}[4 u \cdot \circ] \cdot\right|_{3 \text { cuts }}+\left.\frac{1}{N^{3}}[5 u \cdot \odot]\right|_{3 \text { cuts }}
\end{aligned}
$$

A.2 Couplings in rank $D=4$ tensors models

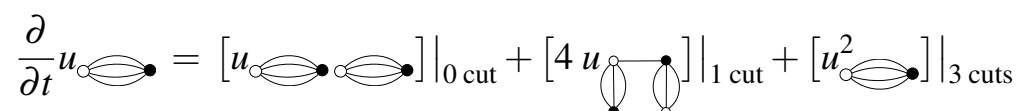

$$
\begin{aligned}
& +\left.\frac{1}{N}\left[6 u \int_{0}\right]\right|_{2 \text { cuts }}+\left.\frac{1}{N^{2}}\left[4 u_{\odot} .\right]\right|_{3 \text { cuts }}+\frac{1}{N^{4}}\left[\left.u_{0} \bullet \bullet \cdot \bullet\right|_{4 \text { cuts }} .\right.
\end{aligned}
$$

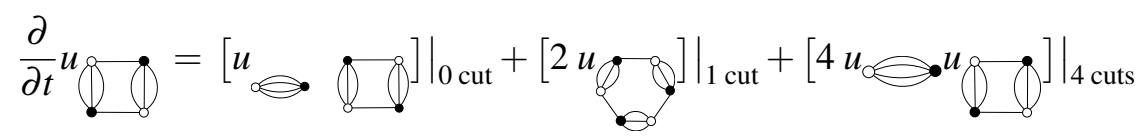

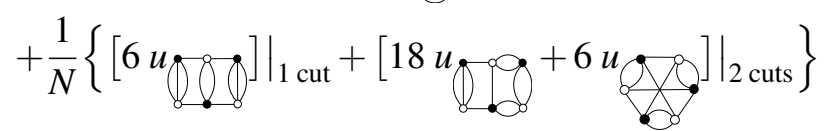

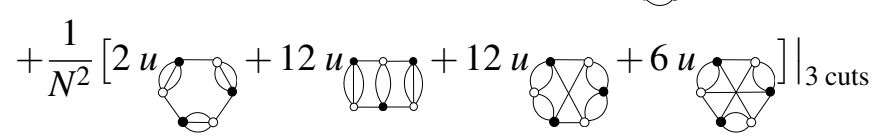

$$
\begin{aligned}
& +\left.\frac{1}{N^{3}}\left[12 u \cdot e_{0}\right]\right|_{4 \text { cuts }}+\frac{1}{N^{4}}\left[\left.4 u_{\ominus} \cdot\right|_{4 \text { cuts }} .\right.
\end{aligned}
$$

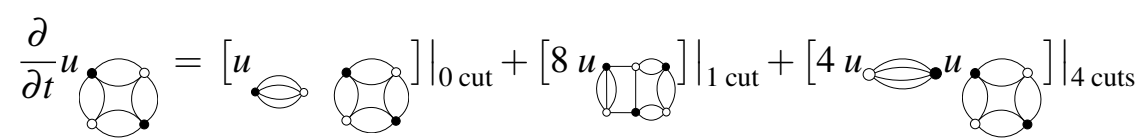

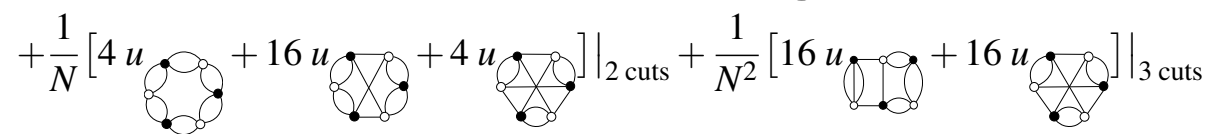

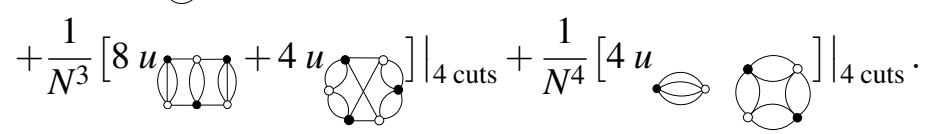




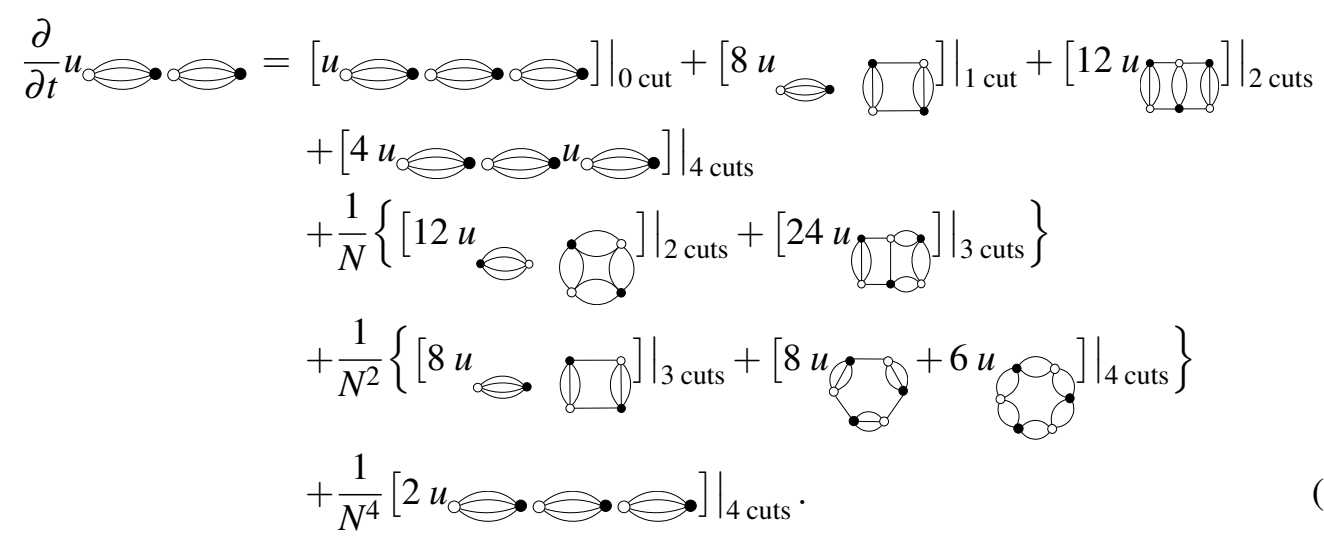

\section{References}

[1] J. Ambjorn, B. Durhuus and T. Jonsson, "Three-dimensional simplicial quantum gravity and generalized matrix models,” Mod. Phys. Lett. A 6 (1991) 1133.

[2] R. Gurau, "Colored Group Field Theory," Commun. Math. Phys. 304 (2011) 69 arXiv:0907.2582 [hep-th].

[3] V. Rivasseau, "The Tensor Track, IV," arXiv:1604.07860 [hep-th]

V. Rivasseau, "The Tensor Track, III," Fortsch. Phys. 62 (2014) 81 [arXiv:1311.1461 [hep-th]].

V. Rivasseau, "The Tensor Track: an Update," arXiv:1209.5284 [hep-th].

V. Rivasseau, "Quantum Gravity and Renormalization: The Tensor Track" AIP Conf. Proc. 1444 (2011) 18 doi:10.1063/1.4715396 arXiv:1112.5104 [hep-th].

[4] R. Gurau, "The complete 1/N expansion of colored tensor models in arbitrary dimensions", Ann.inst.H.Poincaré, 13 399-423, (2012) arXiv:1102.5759 [gr-qc]

[5] R. Gurau, ”Universality for Random Tensors,” Ann. Inst. H. Poincaré Probab. Statist. 50, (2014), 1474 arXiv:1111.0519 [math.PR].

[6] J. Ben Geloun and V. Rivasseau, "A Renormalisable 4-Dimensional Tensor Field Theory," Commun. Math. Phys. 318 (2013) 69 [arXiv:1111.4997 [hep-th]]

[7] S. Carrozza, D. Oriti and V. Rivasseau, "Renormalisation of a SU(2) Tensorial Group Field Theory in Three Dimensions,” Commun. Math. Phys. 330 (2014) 581 [arXiv:1303.6772 [hep-th]].

[8] J. Polchinski, 'Renormalisation and Effective Lagrangians,” Nucl. Phys. B 231 (1984) 269.

[9] T. Krajewski and R. Toriumi, "Polchinski's exact renormalisation group for tensorial theories: Gaussian universality and power counting", arXiv:1511.09084 [gr-qc].

[10] T. Krajewski and R. Toriumi, "Polchinski's equation for group field theory,” Fortsch. Phys. 62 (2014) 855.

[11] T. Krajewski and R. Toriumi, "Exact renormalisation group equations and loop equations for tensor models," arXiv:1603.00172 [gr-qc]. 
[12] V. Bonzom, R. Gurau and V. Rivasseau, "Random tensor models in the large N limit: Uncoloring the colored tensor models,” Phys. Rev. D 85 (2012) 084037 [arXiv:1202.3637 [hep-th]]

[13] J. Zinn-Justin, Quantum field theory theory and critical phenomena Oxford University Press 2002

[14] C. Rovelli Quantum gravity Cambridge University Press 2007

[15] T. Thiemann Modern canonical quantum general relativity Cambridge University Press 2002

[16] L. Freidel, "Group field theory: An Overview" Int. J. Theor. Phys. 44 (2005) 1769-1783. arXiv:hep-th/0505016

[17] T. Krajewski, "Group field theories," PoS QGQGS 2011 (2011) 005 [arXiv:1210.6257 [gr-qc]].

[18] D. Oriti, "Group Field Theory and Loop Quantum Gravity," arXiv:1408.7112 [gr-qc].

[19] D. Benedetti, J. Ben Geloun and D. Oriti, "Functional Renormalisation Group Approach for Tensorial Group Field Theory: a Rank-3 Model,” JHEP 1503 (2015) 084 [arXiv:1411.3180 [hep-th]].

[20] S. Carrozza, "Group field theory in dimension 4- E," Phys. Rev. D 91 (2015) 6, 065023 [arXiv:1411.5385 [hep-th]].

[21] V. Bonzom, T. Delepouve and V. Rivasseau, "Enhancing non-melonic triangulations: A tensor model mixing melonic and planar maps,” Nucl. Phys. B 895 (2015) 161, [arXiv:1502.01365 [math-ph]].

[22] D. O. Samary and F. Vignes-Tourneret, "Just renormalisable TGFT's on U $(1)^{d}$ with Gauge Invariance," Commun. Math. Phys. 329 (2014) 545 [arXiv:1211.2618 [hep-th].

[23] V. Lahoche, D. Oriti and V. Rivasseau, "renormalisation of an Abelian Tensor Group Field Theory: Solution at Leading Order," JHEP 1504 (2015) 095 doi:10.1007/JHEP04(2015)095 arXiv:1501.02086 [hep-th].

[24] D. Benedetti and V. Lahoche, " Functional renormalisation Group Approach for Tensorial Group Field Theory: A Rank-6 Model with Closure Constraint," arXiv:1508.06384 [hep-th]. 